\title{
A PULSED ELLIPTICAL QUADRUPOLE ARRAY FOR TRANSPORT OF MULTIPLE HIGH CURRENT BEAMS
}

\author{
A. Faltens, N.Y. Li, G. Ritchie, D. Shuman*, LBNL, Berkeley,CA
}

\section{Abstract}

We present the design of a pulsed quadrupole magnet array for side by side transport of multiple high current particle beams in induction linear accelerators. The design is directly applicable to low field superconducting quadrupole arrays and can be modified for high fields. The magnetic design minimizes current requirements by placing a modified cosine $2 \theta$ current distribution inside an elliptical iron boundary. The resulting magnet aperture more closely matches the beam's elliptical cross-section, and only the space the beam occupies is magnetized to the required field quality. By eliminating unneeded high field area in the magnet aperture, energy requirements are halved, as compared to an equivalent circular design. The magnet serves double duty as a UHV vacuum chamber/beam tube, and reduced radial thickness results from lower current requirements. Each interior magnet's neighbors serve as the return flux paths and the poles are placed as close as possible to each other to facilitate this. All the above features serve to reduce the beam-to-beam spacing, leading to significant reductions in the size and cost of the induction cores which surround the array, as well as the pulsed power supplies which drive them. The design shown here is for a 21 beam array, yet it is suitable for larger or smaller numbers of beams, as required.

\section{DESIGN OVERVIEW}

The pulsed quadrupole magnet is a high voltage, low inductance, current dominated design, with conductors arranged around an elliptical iron boundary. Figure 1 shows the complete array, and figure 2 shows a cross section view of one quadrupole magnet cell. The magnet's main coil package consists of four 2-layer coils, of 10 turns per coil, 5 turns per layer, epoxy potted onto a thin stainless steel beam tube/end flange assembly, for eddy current minimization. Large, $3.8 \mathrm{~mm}$ diameter insulated copper conductors are used to reduce electrical stress concentrations. Fiberglass cloth and flexible printed circuit correction coils are incorporated into the coil casting for strength and beam steering, respectively. The magnet coil casting carries all magnetic and vacuum loads, both radial and axial.

"Work supported by the Director, Office of Fusion Energy, U.S. DOE, under Contract No. DE-AC03-76SF00098

- email: DBShuman@lbl.gov

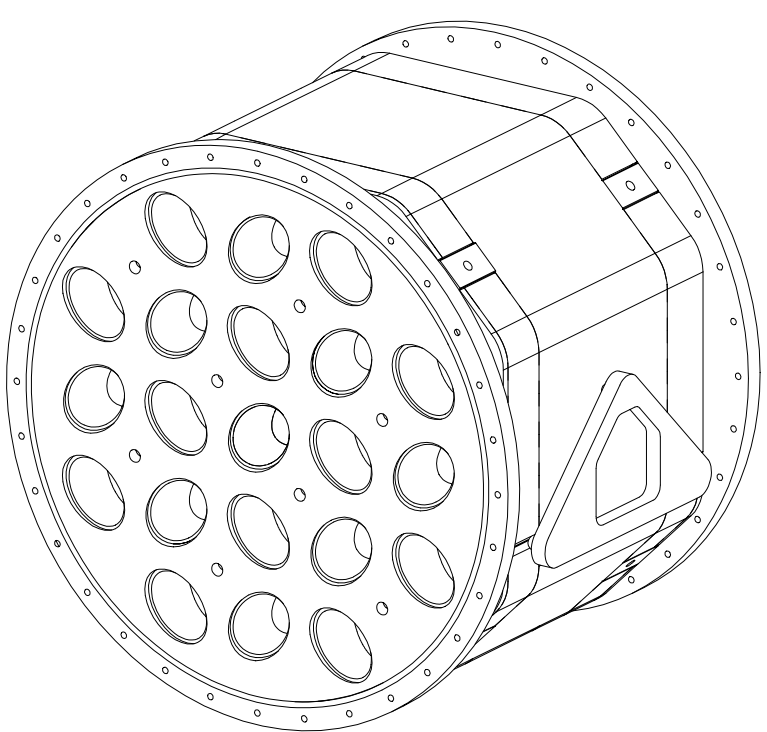

Fig. 1 Complete Quadrupole Array

The laminated iron core serves to position and support the magnets, to provide further reduction in energy requirements by providing a high permeability flux path to the neighboring magnets, to provide a flux return path for the magnets at the edge of the array, and to provide channel isolation for steering coil fluxes.

\section{MAGNETIC DESIGN}

To minimize stored energy, the magnetic field and vacuum volume is confined to an elliptical cross-section,

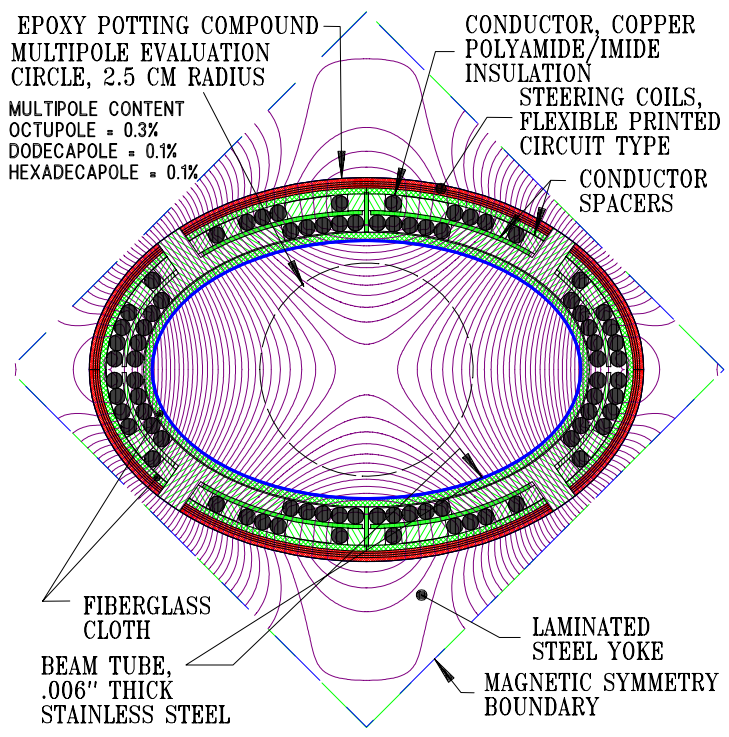

Fig. 2 Quadrupole Cross Section with flux plot 
which corresponds to the beam cross section, with clearance for halo, misalignment, and mis-steering. Conductors are arranged inside an elliptical iron boundary cross section instead of the usual circular one.

Surface current density, $K$, on the boundary follows an analytic function per Faltens, and is proportional to the tangential field component at the iron boundary:

$$
K=K_{0} \frac{a^{2} y^{2}-b^{2} x^{2}}{\sqrt{\left(a^{2} y\right)+\left(b^{2} x\right)}}
$$

Where: $a, b=$ major, minor diameters of elliptical boundary, respectively.

The number of turns is minimized to reduce magnet voltages. The acceptable multipole level at the beam edge determines the minimum number of turns. The conductor size is optimized between reducing eddy currents within the conductor and minimizing field energy outside the beam envelope vs. minimizing $i^{2} \mathrm{R}$ losses and field stresses. Lower losses are possible with finer conductor strands and a larger total conductor volume, but this choice tends to increase the initial stored energy and capacitor cost, and requires higher voltages which compromise magnet longevity. Coil resistive losses are limited to $\sim 10 \%$ of the total energy.

A subsidiary goal is to approximate the conductor current densities $\left(40 \mathrm{kA} / \mathrm{cm}^{2}\right)$ to those that would eventually be used in a superconducting array $\left(30-100 \mathrm{kA} / \mathrm{cm}^{2}\right)$.

\section{Table 1. Magnet Parameters}

\begin{tabular}{|l|l|l|}
\hline Beam Aperture Radius & $5 \times 3$ & $\mathrm{~cm}$ (maj. x min.) \\
\hline Magnet Transverse Pitch & 11.8 & $\mathrm{~cm}$ (horiz., vert.) \\
\hline Magnetic, Total Length & 31,47 & $\mathrm{~cm}$ \\
\hline Field Gradient, B' & 38 & $\mathrm{~T} / \mathrm{m}$ \\
\hline Maximum Field, B & 1.9 & $\mathrm{~T}$ \\
\hline Number of turns & 10 & Turns/coil \\
\hline Conductor diameter & 3.8 & $\mathrm{~mm}$ \\
\hline Magnet Current, I & 4400 & $\mathrm{~A}$ \\
\hline Magnet Resistance, $\mathrm{R}$ & .012 & Ohms \\
\hline Magnet Inductance, $\mathrm{L}$ & .375 & $\mathrm{milliHenries}$ \\
\hline Magnet Voltage, max. & 3 & $\mathrm{kV}(1 \mathrm{mS}$ pulse $)$ \\
\hline Pulse energy/magnet & 3 & $\mathrm{~kJ}$ \\
\hline Energy loss/magnet & 300 & $\mathrm{~J}$ \\
\hline Pulse rate (w/cooling) & 0.5 & $\mathrm{Sec}^{-1}\left(25^{\circ} \mathrm{C} \Delta \mathrm{T}\right)$ \\
\hline
\end{tabular}

To reduce magnet transverse pitch spacing, return flux is routed through adjacent magnets, which allows coils to be placed essentially in contact with each other, as much as practical. Iron is used only to provide the elliptical boundary, to connect the poles in an efficient manner, and to provide for isolation of steering coil flux (skew quadrupole for rotation errors, plus crossed dipoles for positional errors). Higher order multipole corrections are not needed on every lattice period, if needed at all. If so, a separate multipole corrector magnet array will be located after a given number of lattice periods. The magnetic analysis program POISSON/PANDIRA was used, and conductor currents were scaled to account for differing lengths.

\section{COIL DESIGN}

The magnet's short pulse length of $1.0 \mathrm{mS}$ FMFW, requires a high voltage design, with $3 \mathrm{kV}$ between the high voltage lead and ground. As such, electrical stress reduction and good insulation is critical to insure longevity. 7 gauge $(3.8 \mathrm{~mm}$ dia.) copper wire was purchased with a high grade polyamide/imide insulation coating $0.05 \mathrm{~mm}$ thick which tests to $10-15 \mathrm{kV}$ for crossed conductors in a hi-pot test, even after significant bending.

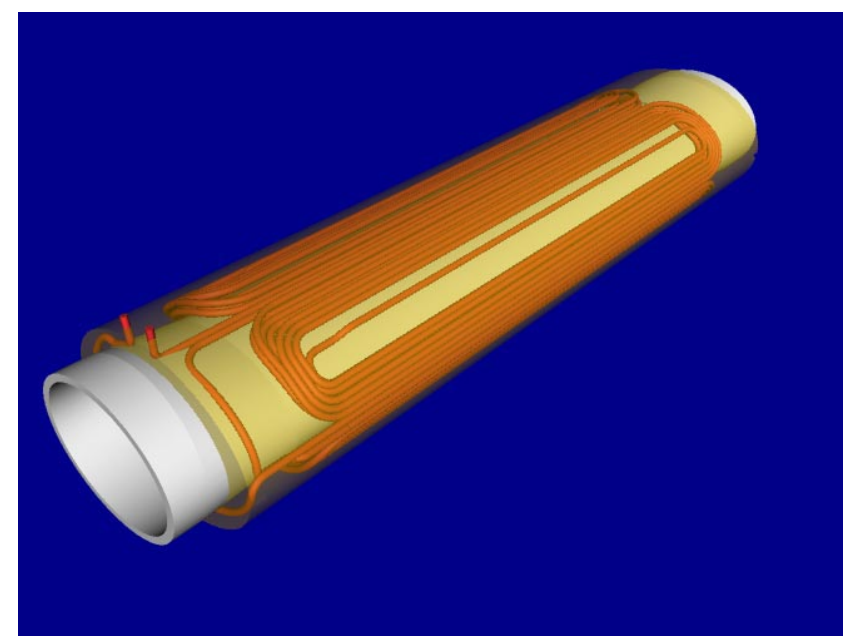

Fig. 3 Coil Casting with Beam Tube and End Flanges

Cooling is by conduction to the iron core, which can be fitted with water cooling channels if necessary. The beam tube/end flange assembly serves as the ground layer on the inner diameter, and a .001" stainless steel foil as the ground layer on the outer diameter (to shield steering coil edges).

\section{VACUUM CHAMBER DESIGN}

To minimize eddy current losses, a stainless steel thin wall tube 0.006" thick is utilized for a beam tube. The beam tube is brazed to end flanges which are flared outwards to allow the beam to expand towards a circular cross-section without beam scraping. To keep the tube from collapsing under vacuum load, it is both restrained by, and bonded to the epoxy potted magnet. The coil casting then carries the vacuum loads, and fiberglass cloth is incorporated in the casting for strength and rigidity. Finite element analysis predicts a maximum vacuum load deflection of $0.04 \mathrm{~mm}$ inward for a potted coil with $0.5 \mathrm{~mm}$ fiberglass layers, both under and over the coils. Maximum stress in the stainless steel beam tube is $5 \mathrm{ksi}$. 


\section{CORE DESIGN}

The core is laminated M36 magnetic steel, .025" thick, with C5 insulation. Each magnet bore extends past the (effective) end of the coil by the average magnet radius of $4 \mathrm{~cm}$ to minimize stray fields. The core will be assembled and held together with insulated stainless steel bolts located between the minor diameters of the magnet bores. The holes between the major diameters are reserved for cooling channels, as heat generation is concentrated in this area. Field is low at the bolt locations, less than 1000G. After coil installation, the entire assembly will be potted in a high melting point wax, which will stabilize the magnets from impulse forces, and allow removal and repair of individual magnets. Magnet bakeout for vacuum purposes is not foreseen to be necessary. The magnet bore edges will be radiused to reduce electrical stress on the leads. Keyways on each magnet bore end align the coils to the core.

\section{ACCELERATOR INTERFACE DESIGN}

Each magnet's end flange features an elliptical cylinder exterior surface for vacuum sealing. The flange inserts into a corresponding aperture in the end manifold plate until the plate rests against the potted end of the magnet. Sealing is accomplished by compressing an O-ring around it using an elliptical O-ring compressor flange, installed from the drift region. This allows easy removal of the manifold plate and magnet repair or replacement, if necessary. Edge clamps or bolts, and perimeter seals are then used to connect the manifold plates to the insulator column edge bellows (not shown). Tie rods running through the core bolts connect the end manifold plates to carry vacuum loads. There is $1 / 2$ " clearance between the manifold plate and the end of the core. Magnet leads exit radially from the coil casting $1 / 4$ " from the end of the coil casting, and exit through this clearance.

\section{PULSER DESIGN}

Two magnets are connected in series to each pulser, which produces $+/-3 \mathrm{kV}$, bipolar. The pulser is an SCR switched capacitive discharge circuit with a recirculator that

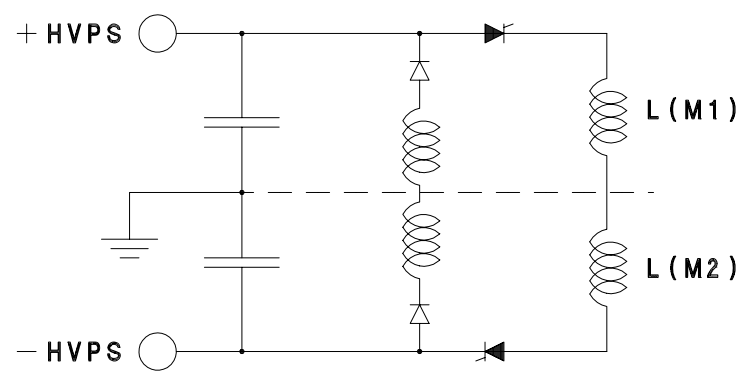

Fig. 4 Pulser Circuit

recovers energy not lost in resistance. For a given rep rate and pulse energy, this lowers the cost of the pulser as well as saves energy. The circuit is shown in fig 4. The components are commercially available except for the choke used in the recirculator, which was fabricated from the same 7 gauge wire as the magnet coils and cast in epoxy.

\section{FABRICATION}

Coils are wound "flat" on a single axis winder, one layer at a time. The individual turns of each layer are wound in an ascending spiral fashion between two plastic dies, one of which is fixed, and the other connected to a movable hydraulic ram. Thus, each wire turn is wound into its own groove, instead of on top of the previous turn, as is normally done. After winding under low tension, the wire ends are clamped off and the coil layer is stretched axially to the point of yield. This removes initial wire curvature and kinks, with negligible reduction in wire diameter or damage to insulation. This operation requires approx. $5001 b f$ for each conductor run.

After winding and stretching, the coil collapses under its own weight into a flat, planar coil layer. Each coil layer is then placed between two plastic dies and pressed to form the elliptical curvature on the ends.

The beam tube/end flange assembly is formed by slipping a round, thin wall stainless steel tube over a threepiece expanding mandrel to form the correct elliptical shape. The end flanges are then low temperature brazed onto each end of the tube.

Magnet potting is done in two steps. First, the mandrel/beam tube/end flange assembly is wrapped with fiberglass cloth, then placed in the potting mold along with four mold inserts. These inserts cast in the central spacers for the bottom coil layers and provide the foundation for coil assembly. The coils and spacers are assembled onto this subassembly. The coils are connected, then wrapped with fiberglass cloth to hold them in place. The foil ground sheet and steering coils, interleaved with fiberglass veil cloth are then wrapped around this subassembly before final potting.

\section{MAGNET PROTOTYPE}

Two single channel quadrupoles were constructed and tested to measure electrical properties of the combined pulser and magnet system, and to verify voltage holding capability. These magnets were connected in series and pulsed $(0.8 \mathrm{mS})$ at $+/-3.2 \mathrm{kV}$ each $(4000 \mathrm{~A})$, at a rate of one pulse every three seconds for 5 hours. After fixing an initial breakdown in one of the leads, the magnets operated successfully with no breakdown or signs of corona. Return voltage available to the recirculator is $82 \%$ of input, or $67 \%$ energy recovery possible. A $20^{\circ} \mathrm{C}$ temperature rise was measured at the end of the test; there was no water or forced air cooling, only thermal conduction to the core, with free convection air cooling. 